\title{
WEAKLY MIXING PROPERTIES OF SEMIGROUPS OF LINEAR OPERATORS
}

\author{
By FUMIO HiAI
}

\section{Introduction.}

The concepts of strong and weak mixing play an important role in the theory of measure-preserving transformations. The strongly mixing condition was connected with the mean ergodic theorem by Blum and Hanson [3], Brunel and Keane [4], and Hanson and Pledger [7], and was generalized to transformations in infinite measure spaces by Krengel and Sucheston [17]. The strongly mixing properties of linear operators of $L_{1}$-spaces have been investigated by $L$ in [18], Akcoglu and Sucheston [1,2], Sato [21], and Fong and Sucheston [6]. On the other hand, the weakly mixing properties have been generalized to linear operators on general Banach spaces in connection with the mean ergodic theorem by Jones $[11,12,13]$, Nagel [20], and Jones and Lin [14].

Let $T$ be a linear operator on a Banach space E. A typical condition meaning strong mixing of $T$ is stated as follows : for each $x \in E, T^{n} x$ converges weakly. A corresponding condition of weak mixing is as follows: for each $x \in E$, there exists a subsequence $\left\{n_{k}\right\}$ of density 1 such that $T^{n_{k}} x$ converges weakly.

In this paper, we shall consider the weakly mixing properties of discrete cyclic semigroups and one parameter semigroups of linear operators on Banach spaces. \& 1 contains some preliminaries concerning upper and lower densities. In $\S 2$ we shall present results concerning the weakly mixing properties of semigroups on Banach spaces. We shall introduce several conditions meaning the weakly mixing properties and including the conditions given in [11], [13], [14], and [20]. Among those conditions, we shall obtain a number of implications. In $\S 3$ we shall give further results for operator convergence of weak mixing type. In $\S 4$ we shall consider semigroups of positive linear operators on $A L$ spaces and strengthen theorems in $\S 3$.

The author would like to express his hearty thanks to Professor H. Umegaki for his constant encouragement and valuable suggestions.

\section{$\S 1$. Preliminaries.}

Let $J$ be a subset of the positive integers $Z^{+}=\{1,2, \cdots\}$, and let $|J|$ denote Received July 13, 1977. 
the cardinality of $J$. The upper density $u d(J)$ and the lower density $l d(J)$ are defined by

$$
\begin{aligned}
& u d(I)=\limsup _{n \rightarrow \infty} n^{-1}|J \cap\{1, \cdots, n\}|, \\
& l d(J)=\liminf _{n \rightarrow \infty} n^{-1}|J \cap\{1, \cdots, n\}|,
\end{aligned}
$$

and if $u d(J)=l d(J)$, the density $d(J)$ by the common limit. Moreover we define the uniform upper density $u d_{*}(J)$ and the unform lower density $l d_{*}(J)$ by

$$
\begin{aligned}
& u d_{*}(J)=\limsup _{n \rightarrow \infty}\left[\sup _{\jmath \geqq 0} n^{-1}|J \cap\{\jmath+1, \cdots, \jmath+n\}|\right], \\
& l d_{*}(J)=\liminf _{n \rightarrow \infty}\left[\inf _{j \geqq 0} n^{-1}|J \cap\{\jmath+1, \cdots, \jmath+n\}|\right],
\end{aligned}
$$

and if $u d_{*}(J)=l d_{*}(J)$, the uniform density $d_{*}(J)$ by the common limit.

Let $A$ be a Lebesgue measurable subset of the positive real numbers $\boldsymbol{R}^{+}=$ $(0, \infty)$, and let $|A|$ denote the Lebesgue measure of $A$. We define the upper density $U D(A)$, the lower density $L D(A)$, the unform upper density $U D_{*}(A)$, and the uniform lower density $L D_{*}(A)$ by

$$
\begin{gathered}
U D(A)=\limsup _{t \rightarrow \infty} t^{-1}|A \cap(0, t]|, \\
L D(A)=\liminf t_{t \rightarrow \infty}^{-1}|A \cap(0, t]|, \\
U D_{*}(A)=\lim \sup _{t \rightarrow \infty}\left[\sup _{r \geq 0} t^{-1}|A \cap(r, r+t]|\right], \\
L D_{*}(A)=\liminf _{t \rightarrow \infty}\left[\inf _{r \geq 0} t^{-1}|A \cap(r, r+t]|\right] .
\end{gathered}
$$

If $U D(A)=L D(A)$, we define the density $D(A)$ by the common limit, and also if $U D_{*}(A)=L D_{*}(A)$, the uniform density $D_{*}(A)$ by the common limit. Throughout this paper, subsets of $\boldsymbol{R}^{+}$are always assumed to be Lebesgue measurable.

The formulas $0 \leqq l d_{*}(J) \leqq l d(J) \leqq u d(J) \leqq u d_{*}(J) \leqq 1, \quad l d(J)=1-u d\left(\boldsymbol{Z}^{+} \backslash J\right)$ and $l d_{*}(J)=1-u d_{*}\left(\boldsymbol{Z}^{+} \backslash J\right)$ are immediately verified. Note that there is a $J \subset \boldsymbol{Z}^{+}$such that $u d(J)=0$ but $u d_{*}(J)=1$. The same formulas hold for densities in $\boldsymbol{R}^{+}$.

The following lemma is well known and easily proved (cf. [13], [24, p. 40]).

LEMmA 1.1. ( $\left.1^{\circ}\right)$ Let $\left\{J_{k}\right\}$ be a sequence of subsets of $\boldsymbol{Z}^{+}$with $d\left(J_{k}\right)=1$.

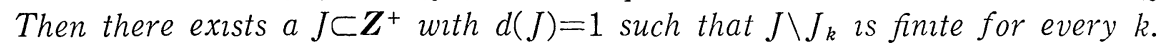

$\left(2^{\circ}\right)$ Let $\left(\alpha_{n}\right)$ be a bounded sequence of real numbers. Then $\lim _{n \rightarrow \infty} n^{-1} \sum_{i=1}^{n}\left|\alpha_{i}\right|=0$ if and only if there exists a $J \subset Z^{+}$with $d(J)=1$ such that $\alpha_{n} \rightarrow 0$ as $n \in J, n \rightarrow \infty$.

In the same way, we have the following:

Lemma 1.2. ( $\left(1^{\circ}\right)$ Let $\left\{A_{k}\right\}$ be a sequence of subsets of $\boldsymbol{R}^{+}$with $D\left(A_{k}\right)=1$. Then there exists an $A \subset \boldsymbol{R}^{+}$with $D(A)=1$ such that $A \backslash A_{k}$ is bounded for every $k$. 
$\left(2^{\circ}\right)$ Let $\phi$ be a Lebesgue measurable and bounded real function on $\boldsymbol{R}^{+}$. Then $\lim _{t \rightarrow \infty} t^{-1} \int_{0}^{t}|\phi(s)| d s=0$ if and only if there exists an $A \subset \boldsymbol{R}^{+}$with $D(A)=1$ such that $\phi(t) \rightarrow 0$ as $t \in A, t \rightarrow \infty$.

Lemma 1.3. Let $A \subset \boldsymbol{R}^{+}$and $\delta>0$ be given and let

$$
J=\left\{n \in \boldsymbol{Z}^{+}:|A \cap((n-1) \delta, n \delta]|>0\right\} .
$$

Then $u d(J) \geqq U D(A), l d(J) \geqq L D(A), u d_{*}(J) \geqq U D_{*}(A)$, and $l d_{*}(J) \geqq L D_{*}(A)$. have

Proof. If $j \delta \leqq r<(j+1) \delta$ and $n \delta \leqq t<(n+1) \delta$ where $j \geqq 0$ and $n \geqq 1$, then we

$$
\begin{aligned}
t^{-1}|A \cap(r, r+t]| & \leqq(n \delta)^{-1}|A \cap(j \delta,(j+n+2) \delta]| \\
& \leqq(n \delta)^{-1} \delta|J \cap\{j+1, \cdots, j+n+2\}| \\
& \leqq n^{-1}[|J \cap\{j+1, \cdots, j+n\}|+2] .
\end{aligned}
$$

Thus the desired inequalities are obvious.

Q.E.D.

The following lemma is a slight extension of Jones [11, Lemma 3], and we shall give the proof only substituting the uniform upper density for the upper density in Jones' proof.

Lemma 1.4. Let $J, K$ be subsets of $Z^{+}$with $u d(J)=1, u d_{*}(K)>0$. Then there exists an infinite subset $\left\{p_{i}\right\}$ of $J$ such that any finte subset of $\left\{p_{i}\right\}$ is translated infinitely often in $K$, i.e., for each $n$,

$$
\left\{p_{1}, \cdots, p_{n}\right\}+\{k\}=\left\{p_{1}+k, \cdots, p_{n}+k\right\} \subset K
$$

for infinitely many $k$.

Proof. Let $u d_{*}(K)=\alpha>0$. Choose an integer $N$ so large that $N^{-1}<\alpha / 3$ and $N^{-1}\left|\left(Z^{+} \backslash J\right) \cap\{1, \cdots, N\}\right|<\alpha / 3$. Let $K_{p}=\{k \in K: k+p \in K\}$ for $p \in J \cap\{1, \cdots, N\}$, and $K_{0}=K \backslash \bigcup_{p \in J \cap\{1, \cdots, N\}} K_{p}$. If $k \in K_{0}$, then we have

$$
|K \cap\{k+1, \cdots, k+N\}| \leqq\left|\left(\boldsymbol{Z}^{+} \backslash J\right) \cap\{1, \cdots, N\}\right| .
$$

Thus it follows that

$$
\left|K_{0} \cap\{k+1, \cdots, k+N\}\right| \leqq 1+\left|\left(\boldsymbol{Z}^{+} \backslash J\right) \cap\{1, \cdots, N\}\right|, \quad k \geqq 0 .
$$

If $m N<n \leqq(m+1) N$, then we have

$$
\begin{aligned}
& n^{-1}\left|K_{0} \cap\{k+1, \cdots, k+n\}\right| \\
& \quad \leqq \frac{m+1}{m}\left[N^{-1}+N^{-1}\left|\left(\boldsymbol{Z}^{+} \backslash J\right) \cap\{1, \cdots, N\}\right|\right], \quad k \geqq 0 .
\end{aligned}
$$

Hence we obtain $u d_{*}\left(K_{0}\right) \leqq 2 \alpha / 3$. Since 


$$
u d_{*}(K) \leqq u d_{*}\left(K_{0}\right)+\sum_{p \in J \cap i 1, \ldots, N\}} u d_{*}\left(K_{p}\right),
$$

there exists a $p_{1} \in J \cap\{1, \cdots, N\}$ such that $\left\{0, p_{1}\right\}+K^{1} \subset K$ and $u d_{*}\left(K^{1}\right)>0$ where $K^{1}=K_{p_{1}}$. Now substituting $K^{1}$ for $K$ and $J \backslash\left\{1, \cdots, p_{1}\right\}$ for $J$ in the above argument, we get $\left\{0, p_{2}\right\}+K^{2} \subset K^{1}$ with $p_{1}<p_{2} \in J$ and $u d_{*}\left(K^{2}\right)>0$, and so $\left\{0, p_{1}, p_{2}\right\}$ $+K^{2} \subset K$. Repeating indefinitely, we obtain a sequence $p_{1}<p_{2}<\cdots$ in $J$ such that $\left\{0, p_{1}, \cdots, p_{n}\right\}+K^{n} \subset K$ where $K^{n}$ is infinite with $u d_{*}\left(K^{n}\right)>0$.

Let $\left(\alpha_{n k}\right)_{n, k \geq 1}$ be a matrix of real numbers. We shall need the following conditions :

$$
\begin{aligned}
& \sup _{n} \sum_{k}\left|\alpha_{n k}\right|<\infty, \\
& \lim _{n \rightarrow \infty} \sum_{k} \alpha_{n k}=1, \\
& \lim _{n \rightarrow \infty} \sum_{k \in K}\left|\alpha_{n k}\right|=0 \text { for any } K \subset Z^{+} \text {with } d(K)=0, \\
& \lim _{n \rightarrow \infty} \sum_{k \in K}\left|\alpha_{n k}\right|=0 \text { for any } K \subset Z^{+} \text {with } d_{*}(K)=0 .
\end{aligned}
$$

Similarly, for a sequence $\left\{\phi_{n}\right\}$ of Lebesgue measurable real functions on $\boldsymbol{R}^{+}$, we shall need the following conditions:

$$
\begin{aligned}
& \sup _{n} \int_{0}^{\infty}\left|\phi_{n}(t)\right| d t<\infty, \\
& \lim _{n \rightarrow \infty} \int_{0}^{\infty} \phi_{n}(t) d t=1, \\
& \lim _{n \rightarrow \infty} \int_{B}\left|\phi_{n}(t)\right| d t=0 \text { for any } B \subset \boldsymbol{R}^{+} \text {with } D(B)=0, \\
& \lim _{n \rightarrow \infty} \int_{B}\left|\phi_{n}(t)\right| d t=0 \text { for any } B \subset \boldsymbol{R}^{+} \text {with } D_{*}(B)=0 .
\end{aligned}
$$

For example, in connection with abelian ergodic theorem, a sequence $\left\{\phi_{n}\right\}$ defined by $\phi_{n}(t)=\lambda_{n} e^{-\lambda_{n} t}$ with $\lambda_{n} \downarrow 0$ satisfies the above (1.5)-(1.7).

\section{$\S 2$. Semigroups on Banach spaces (1).}

Let $E$ be a real or complex Banach space with the dual space $E^{*}$, and denote by $\mathcal{L}(E)$ the space of all bounded linear operators on $E$. We shall first establish several conditions corresponding to weak mixing of discrete cyclic semigroups in $\mathcal{L}(E)$. Let $T \in \mathcal{L}(E)$. For each $x \in E$, we consider the following conditions :

(i) ${ }_{1}$ there exists a $J \subset Z^{+}$with $d(J)=1$ such that $T^{n} x$ converges weakly to 0 as $n \in J, n \rightarrow \infty$;

(ii) $)_{1}$ for each matrix $\left(\alpha_{n k}\right)$ with (1.1) and (1.4), $\sum_{k=1}^{\infty} \alpha_{n k} T^{k} x$ converges strongly 
to 0 as $n \rightarrow \infty$;

(iii) ${ }_{1}$ for each matrix $\left(\alpha_{n k}\right)$ with (1.1) and (1.3), $\sum_{k=1}^{\infty} \alpha_{n k} T^{k} x$ converges strongly to 0 as $n \rightarrow \infty$;

(iv) ${ }_{1}$ for each $K \subset Z^{+}$with $l d_{*}(K)>0$, the averages

$$
|K \cap\{j+1, \cdots, j+n\}|^{-1} \sum_{k \in K \cap(j+1, \cdots, j+n\}} T^{k} x
$$

converge strongly to 0 as $n \rightarrow \infty$ uniformly with respect to $j \geqq 0$;

$(\mathrm{v})_{1}$ for each $K \subset Z^{+}$with $l d(K)>0$, the averages

$$
|K \cap\{1, \cdots, n\}|^{-1} \sum_{k \in K \cap(1, \cdots, n\}} T^{k} x
$$

converge strongly to 0 as $n \rightarrow \infty$;

(vi)

$$
\lim _{n \rightarrow \infty} \sup _{\substack{\left\|x^{*}\right\| \leq 1 \\ j \geq 0}} n^{-1} \sum_{k=j+1}^{j+n}\left|\left\langle T^{k} x, x^{*}\right\rangle\right|=0
$$

(vii) ${ }_{1}$ for each $x^{*} \in E^{*}$,

$$
\lim _{n \rightarrow \infty} n^{-1} \sum_{k=1}^{n}\left|\left\langle T^{k} x, x^{*}\right\rangle\right|=0
$$

(viii) $)_{1}$ the weak closure of $\left\{T^{n} x: n \in Z^{+}\right\}$contans 0 .

Over these conditions, we present the following :

THEOREM 2.1. Let $T \in \mathcal{L}(E)$ and assume that $\sup \left\{\left\|T^{n}\right\|: n \in \boldsymbol{Z}^{+}\right\}<\infty$. Then, for each $x \in E$, the following statements $\left(1^{\circ}\right)$ and $\left(2^{\circ}\right)$ hold:

$\left(1^{\circ}\right) \quad(\mathrm{i})_{1} \Rightarrow(\mathrm{ii})_{1} \Rightarrow(\mathrm{iii})_{1} \Rightarrow(\mathrm{iv})_{1} \Leftrightarrow(\mathrm{v})_{1} \Leftrightarrow(\mathrm{vi})_{1} \Leftrightarrow(\mathrm{vii})_{1} \Rightarrow(\mathrm{viii})_{1}$.

$\left(2^{\circ}\right)$ If the weak closure of $\left\{T^{n} x: n \in \boldsymbol{Z}^{+}\right\}$is metrizable in the weak topology (in particular, if $\left\{T^{n} x: n \in \boldsymbol{Z}^{+}\right\}$is weakly sequentially compact), then all the conditions $(\mathrm{i})_{1}-(\text { viii })_{1}$ are equivalent.

We shall next consider the case of one parameter semigroups in $\mathcal{L}(E)$. Let $\left\{T(t): t \in \boldsymbol{R}^{+}\right\}$be a strongly measurable and uniformly bounded semigroup in $\mathcal{L}(E)$. For each $x \in E$, we consider the following conditions:

(I) ${ }_{1}$ there exists an $A \subset \boldsymbol{R}^{+}$with $D(A)=1$ such that $T(t) x$ converges weakly to 0 as $t \in A, t \rightarrow \infty$;

$(\mathrm{II})_{1}$ for each sequence $\left\{\dot{\phi}_{n}\right\}$ with (1.5) and (1.8), $\int_{0}^{\infty} \phi_{n}(s) T(s) x d s$ converges strongly to 0 as $n \rightarrow \infty$;

(III) $)_{1}$ for each sequence $\left\{\phi_{n}\right\}$ with (1.5) and (1.7), $\int_{0}^{\infty} \phi_{n}(s) T(s) x d s$ converges strongly to 0 as $n \rightarrow \infty$;

$(\mathrm{IV})_{1}$ for each $B \subset \boldsymbol{R}^{+}$with $L D_{*}(B)>0$, the averages 


$$
|B \cap(r, r+t]|^{-1} \int_{B \cap(r, r+t]} T(s) x d s
$$

converge strongly to 0 as $t \rightarrow \infty$ unformly with respect to $r \geqq 0$;

$(\mathrm{V})_{1}$ for each $B \subset \boldsymbol{R}^{+}$with $L D(B)>0$, the averages

$$
|B \cap(0, t]|^{-1} \int_{B \cap(0, t]} T(s) x d s
$$

converge strongly to 0 as $t \rightarrow \infty$;

$(\mathrm{VI})_{1}$

$$
\lim _{t \rightarrow \infty} \sup _{\substack{\left\|, x^{*}\right\| \leq 1 \\ r \geq 0}} t^{-1} \int_{r}^{r+t}\left|\left\langle T(s) x, x^{*}\right\rangle\right| d s=0 ;
$$

$(\mathrm{VII})_{1}$ for each $x^{*} \in E^{*}$,

$$
\lim _{t \rightarrow \infty} t^{-1} \int_{0}^{t}\left|\left\langle T(s) x, x^{*}\right\rangle\right| d s=0 ;
$$

$(\mathrm{VIII})_{1}$ the weak closure of $\left\{T(t) x: t \in \boldsymbol{R}^{+}\right\}$contains 0 .

Over these conditions, we present the following:

THEOREM 2.2. Let $\left\{T(t): t \in \boldsymbol{R}^{+}\right\}$be a strongly measurable and uniformly bounded semigroup in $\mathcal{L}(E)$. Then, for each $x \in E$, the following statements $\left(1^{\circ}\right)$ and $\left(2^{\circ}\right)$ hold:

$\left(1^{\circ}\right) \quad(\mathrm{I})_{1} \Rightarrow(\mathrm{II})_{1} \Leftrightarrow(\mathrm{III})_{1} \Leftrightarrow(\mathrm{IV})_{1} \Leftrightarrow(\mathrm{V})_{1} \Leftrightarrow(\mathrm{VI})_{1} \Leftrightarrow(\mathrm{VII})_{1} \Rightarrow(\mathrm{VIII})_{1}$.

$\left(2^{\circ}\right)$ If the weak closure of $\{T(t) x: t \geqq r\}$ is metrizable in the weak topology for some $r>0$ (in particular, if $\{T(t) x: t \geqq r\}$ is weakly sequentially compact for some $r>0)$, then all the conditions $(\mathrm{I})_{1}-(\mathrm{VIII})_{1}$ are equivalent.

We note that some parts of the above theorems have been already proved as stated in Remarks (1)-(3) below.

REMARKS. (1) Jones [11] called $T \in \mathcal{L}(E)$ weakly mixing at $x$ if the condition $(\mathrm{i})_{1}$ holds. The implication $(\mathrm{i})_{1} \Rightarrow(\mathrm{v})_{1}$ is the main result in [11]. The equivalence of (i) and (vii) $)_{1}$ was given in [13] under the assumption of $\left\{T^{n} x: n \in \boldsymbol{Z}^{+}\right\}$ being weakly sequentially compact.

(2) If $x \in E$ satisfies the condition (viii) $)_{1}$ or (VIII) $)_{1}$, then $x$ is called to be a flight vector (cf. [10, p. 22]). Applying the deLeeuw-Glicksberg theory [5], Junghenn [15] presented the equivalence of $(\mathrm{I})_{1},(\mathrm{VII})_{1}$ and (VIII) ${ }_{1}$ for a one parameter weakly almost periodic equicontinuous semigroup of class $C_{0}$ acting on a locally convex linear topological space.

(3) Jones and Lin [14] proved the equivalence of $(\mathrm{v})_{1},(\mathrm{vi})_{1}$ and (vii) $)_{1}$, and also improved the result in [11] without the use of the combinatorial machinery of $[11]$.

(4) The conditions (ii) ${ }_{1}$ and (iii) $)_{1}$ are considered to be the weakly mixing versions of the condition given in $[6,2]$.

(5) In Theorem 2.2, if the weak closure of $\{T(t) x: t \geqq r\}$ is metrizable [or 
resp., compact] in the weak topology for some $r>0$, then so is the weak closure of $\{T(t) x: t \geqq s\}$ for every $s>0$.

In the remainder of this section, we shall give the proof of Theorem 2.2. Since Theorem 2.1 can be proved analogously and more easily, we shall omit the details for it.

LEMMA 2.3. If $X$ is a weakly compact and strongly separable subset of $E$, then $X$ is metrizable in the weak topology.

Proof. Take a sequence $\left\{y_{n}\right\}$ which is dense in $X-X=\{x-y: x, y \in X\}$. For each $n$, choose a $y_{n}^{*} \in E^{*}$ such that $\left\|y_{n}^{*}\right\|=1$ and $\left\langle y_{n}, y_{n}^{*}\right\rangle=\left\|y_{n}\right\|$, and define

$$
d(x, y)=\sum_{n=1}^{\infty} 2^{-n}\left|\left\langle x-y, y_{n}^{*}\right\rangle\right|, \quad x, y \in X .
$$

Then it is easy to see that $d$ is a metric on $X$ whose topology is weaker than the weak topology. Thus these topologies on $X$ are identical.

Q.E.D.

Proof of Theorem 2.2. (I $)_{1} \Rightarrow(\mathrm{II})_{1}$. Let (II) $)_{1}$ be false. Then there exists a sequence $\left\{\phi_{n}\right\}$ with (1.5) and (1.8) such that $\left\|\int_{0}^{\infty} \phi_{n}(s) T(s) x d s\right\| \geqq \varepsilon>0$ for all $n$. Taking $\max \left(\phi_{n}, 0\right)$ or $\max \left(-\phi_{n}, 0\right)$ instead of $\phi_{n}$, we may assume that every $\phi_{n}$ is nonnegative. Let $M=\sup \left\{\|T(t)\|: t \in R^{+}\right\}$. In view of (1.8), we can choose a subsequence $\left\{\phi_{n}\right\}$ of $\left\{\phi_{n}\right\}$ and a sequence $r_{1}<t_{1}<r_{2}<t_{2}<\cdots$ such that

$$
\begin{gathered}
\int_{0}^{r_{n}} \psi_{n}(s) d s+\int_{t_{n}}^{\infty} \psi_{n}(s) d s<\frac{\varepsilon}{2 M\|x\|}, \\
r_{n+1}>t_{n}+n .
\end{gathered}
$$

Then it follows from (2.1) that $\left\|\int_{r_{n}}^{t_{n}} \psi_{n}(s) T(s) x d s\right\| \geqq \varepsilon / 2$ for all $n$. For each $n$, choosing an $x_{n}^{*} \in E^{*}$ with $\left\|x_{n}^{*}\right\|=1$ such that

$$
\int_{r_{n}}^{t_{n}} \psi_{n}(s) \operatorname{Re}\left\langle T(s) x, x_{n}^{*}\right\rangle d s \geqq \frac{\varepsilon}{2},
$$

we define

$$
B_{n}=\left\{s: s \in\left(r_{n}, t_{n}\right], \operatorname{Re}\left\langle T(s) x, x_{n}^{*}\right\rangle \geqq \beta\right\},
$$

where $0<\beta<\varepsilon /\left(4 \sup _{n} \int_{0}^{\infty} \psi_{n}(s) d s\right)$. Then we have

$$
\begin{aligned}
\frac{\varepsilon}{2} \leqq & \int_{B_{n}} \psi_{n}(s) \operatorname{Re}\left\langle T(s) x, x_{n}^{*}\right\rangle d s \\
& +\int_{\left(r_{n}, t_{n} \backslash B_{n}\right.} \psi_{n}(s) \operatorname{Re}\left\langle T(s) x, x_{n}^{*}\right\rangle d s \\
\leqq & M\|x\| \int_{B_{n}} \psi_{n}(s) d s+\beta \int_{0}^{\infty} \psi_{n}(s) d s,
\end{aligned}
$$


so that $\int_{B_{n}} \psi_{n}(s) d s \geqq \varepsilon /(4 M\|x\|)$ for all $n$. Thus, putting $B=\bigcup_{n=1}^{\infty} B_{n}$, we obtain $U D_{*}(B)>0$ by (1.8). Now suppose that (I) holds, and let $A \subset \boldsymbol{R}^{+}$be a set taken in $(\mathrm{I})_{1}$. Since $T(t) x$ is strongly continuous in $\boldsymbol{R}^{+}(\mathrm{cf} .[9, \mathrm{p} .305])$ and hence uniformly strongly continuous in $t \geqq 1$, there exists a $\delta>0$ such that $\|T(u) x-T(v) x\|$ $<\beta /(2 M)$ for $u, v \geqq 1,|u-v|<\delta$. Define

$$
\begin{aligned}
& J=\{n:|A \cap((n-1) \delta, n \delta]|>0\}, \\
& K=\{n:|B \cap((n-1) \delta, n \delta]|>0\} .
\end{aligned}
$$

Then it follows from Lemma 1.3 that $d(J)=D(A)=1$ and $u d_{*}(K) \geqq U D_{*}(B)>0$. By Lemma 1.4, there exists a sequence $\left\{p_{i}\right\} \subset J$ with $1+\delta^{-1}<p_{1}<p_{2}<\cdots$ such that any finite subset of $\left\{p_{i}\right\}$ is translated infinitely often in $K$. Taking $u_{\imath}=p_{i} \delta$ $-s_{i} \in A$ where $0 \leqq s_{\imath}<\delta$, since $T\left(u_{\imath}\right) x \rightarrow 0$ (weakly), we can choose $\alpha_{1}, \cdots, \alpha_{N} \geqq 0$ with $\sum_{i=1}^{N} \alpha_{i}=1$ such that $\left\|\sum_{i=1}^{N} \alpha_{\imath} T\left(u_{\imath}\right) x\right\|<\beta /(2 M)$. In view of (2.2), if we take a sufficiently large $k$ with $\left\{p_{1}+k, \cdots, p_{N}+k\right\} \subset K$, then it follows that there exists some $n_{0}$ for which $\left(p_{i}+k\right) \delta-t_{i} \in B_{n_{0}}$ where $0 \leqq t_{2}<\delta, 1 \leqq i \leqq N$. Thus we obtain $\left\|\sum_{i=1}^{N} \alpha_{\imath} T\left(v_{i}+k \delta\right) x\right\| \geqq \beta$ by the definition of $B_{n_{0}}$ where $v_{i}=p_{i} \delta-t_{\imath}$. On the other hand, since $u_{\imath}, v_{i} \geqq 1$ and $\left|u_{i}-v_{i}\right|<\delta$, we have

$$
\begin{aligned}
& \left\|\sum_{\imath=1}^{N} \alpha_{\imath} T\left(v_{i}+k \delta\right) x\right\| \leqq M\left\|\sum_{\imath=1}^{N} \alpha_{\imath} T\left(v_{\imath}\right) x\right\| \\
& \leqq M\left\{\left\|\sum_{\imath=1}^{N} \alpha_{\imath} T\left(u_{\imath}\right) x\right\|+\left\|\sum_{\imath=1}^{N} \alpha_{\imath}\left(T\left(u_{\imath}\right)-T\left(v_{i}\right)\right) x\right\|\right\}<\beta,
\end{aligned}
$$

which is a contradiction.

The implication (II) $)_{1} \Rightarrow(\text { III })_{1}$ is trivial.

$(\mathrm{III})_{1} \Rightarrow(\mathrm{V})_{1}$. Let $B \subset \boldsymbol{R}^{+}$with $L D(B)>0$ and $t_{n} \uparrow \infty$ be given. We may assume that $\left|B \cap\left(0, t_{n}\right]\right|>0$ for all $n$. Define a sequence $\left\{\phi_{n}\right\}$ by $\phi_{n}(s)=$ $\left|B \cap\left(0, t_{n}\right]\right|^{-1}$ if $s \in B \cap\left(0, t_{n}\right]$, and $\phi_{n}(s)=0$ otherwise. Then $\left\{\phi_{n}\right\}$ satisfies (1.5) and (1.7). Therefore (III) $)_{1}$ implies that

$$
\int_{0}^{\infty} \phi_{n}(s) T(s) x d s=\left|B \cap\left(0, t_{n}\right]\right|^{-1} \int_{B \cap\left(0, t_{n}\right]} T(s) x d s
$$

converges strongly to 0 as $n \rightarrow \infty$.

$(\mathrm{V})_{1} \Rightarrow(\mathrm{VII})_{1}$. Let (VII $)_{1}$ be false. Then there exist $x^{*} \in E^{*}$ with $\left\|x^{*}\right\|=1$, $\varepsilon>0$ and $t_{n} \uparrow \infty$ such that

Putting

$$
t_{n}{ }^{-1} \int_{0}^{t_{n}}\left|\left\langle T(s) x, x^{*}\right\rangle\right| d s \geqq \varepsilon, \quad n \geqq 1 .
$$

$$
B=\left\{s:\left|\left\langle T(s) x, x^{*}\right\rangle\right| \geqq \frac{\varepsilon}{2}\right\},
$$


we have

$$
t_{n}{ }^{-1}\left|B \cap\left(0, t_{n}\right]\right| \geqq \frac{\varepsilon}{2 M\|x\|}, \quad n \geqq 1,
$$

so that $U D(B)>0$. Thus, replacing $x^{*}$ by $-x^{*}$ or $i x^{*}$ or $-i x^{*}(i=\sqrt{-1})$ if necessary, we obtain $U D\left(B^{\prime}\right)>0$ where

$$
B^{\prime}=\left\{s: \operatorname{Re}\left\langle T(s) x, x^{*}\right\rangle \geqq \frac{\varepsilon}{4}\right\} .
$$

Let $0<\beta<U D\left(B^{\prime}\right)$ and $0<\delta<\varepsilon \beta /(4 M\|x\|)$, and let $C=B^{\prime} \bigcup_{k=1}^{\infty}(k, k+\delta]$. Then, for $t \in \boldsymbol{R}^{+}$with $t^{-1}\left|B^{\prime} \cap(0, t]\right|>\beta$, we have

$$
\begin{aligned}
& \int_{C \cap(0, t]} \operatorname{Re}\left\langle T(s) x, x^{*}\right\rangle d s \\
& \geqq \int_{B^{\prime} \cap(0, t]} \operatorname{Re}\left\langle T(s) x, x^{*}\right\rangle d s \\
& \quad-\left|\int_{\left(C \backslash B^{\prime}\right) \cap(0, t]} \operatorname{Re}\left\langle T(s) x, x^{*}\right\rangle d s\right| \\
& \geqq \frac{\varepsilon}{4}\left|B^{\prime} \cap(0, t]\right|-M\|x\| \delta t \\
& \geqq\left(\frac{\varepsilon \beta}{4}-M\|x\| \delta\right) t \\
& \geqq\left(\frac{\varepsilon \beta}{4}-M\|x\| \delta\right)|C \cap(0, t]| .
\end{aligned}
$$

Since $L D(C)>0$, this shows that $(\mathrm{V})_{1}$ fails to hold.

$(\mathrm{VII})_{1} \Rightarrow(\mathrm{VI})_{1}$. We first assume that $\sup \left\{\|T(t)\|: t \in \boldsymbol{R}^{+}\right\} \leqq 1$. Let $K=$ $\left\{x^{*} \in E^{*}:\left\|x^{*}\right\| \leqq 1\right\}$ which is a compact Hausdorff space in the weak* topology. Since $T(t)^{*} K \subset K$ and $T(t)^{*}$ is continuous on $K$ for all $t \in \boldsymbol{R}^{+}$, we can define a one parameter semigroup $\left\{\hat{T}(t): t \in \boldsymbol{R}^{+}\right\}$of Markov operators on $C(K)$, the Banach space of all continuous functions on $K$ with the supremum norm, by $(\hat{T}(t) f)\left(x^{*}\right)$ $=f\left(T(t)^{*} x^{*}\right), x^{*} \in K, f \in C(K)$. Note that $\hat{T}(t)$ is continuous in the weak operator topology and hence in the strong operator topology (cf. [9, p. 306]). Let $f\left(x^{*}\right)$ $=\left|\left\langle x, x^{*}\right\rangle\right|$. Then, for $\mu \in C(K)^{*}$ such that $\hat{T}(t)^{*} \mu=\mu$ for all $t \in \boldsymbol{R}^{+}$, we have

$$
\langle f, \mu\rangle=\langle\hat{T}(s) f, \mu\rangle=\int_{K}\left|\left\langle T(s) x, x^{*}\right\rangle\right| d \mu\left(x^{*}\right), \quad s \in \boldsymbol{R}^{+} .
$$

Hence, by Fubini's theorem, we have

$$
\langle f, \mu\rangle=\int_{K}\left(t^{-1} \int_{0}^{t}\left|\left\langle T(s) x, x^{*}\right\rangle\right| d s\right) d \mu\left(x^{*}\right),
$$


so that $\langle f, \mu\rangle=0$ by (VII) $)_{1}$. This shows that $f$ is contained in the closed subspace spanned by $\bigcup_{t \in R^{+}}(I-\hat{T}(t)) C(K)$, and hence $t^{-1} \int_{0}^{t} \hat{T}(s) f d s$ converges strongly to 0 as $t \rightarrow \infty$. Thus we have

$$
\begin{aligned}
& \sup _{\substack{\left\|, x^{*}\right\| \leq 1 \leq 1 \\
r \geq 0}} t^{-1} \int_{r}^{r+t}\left|\left\langle T(s) x, x^{*}\right\rangle\right| d s \\
& =\sup _{r \geq 0}\left\|t^{-1} \int_{r}^{r+t} \hat{T}(s) f d s\right\| \\
& =\sup _{r \geqq 0}\left\|\hat{T}(r)\left(t^{-1} \int_{0}^{t} \hat{T}(s) f d s\right)\right\| \\
& \leqq\left\|t^{-1} \int_{0}^{t} \hat{T}(s) f d s\right\| \longrightarrow 0
\end{aligned}
$$

For the case when $\sup \left\{\|T(t)\|: t \in \boldsymbol{R}^{+}\right\}<\infty$, we define an equivalent norm on $E$ by

$$
\|x\|=\max \left\{\|x\|, \sup _{t \in R^{+}}\|T(t) x\|\right\} .
$$

Since $\sup \left\{\|T(t)\| \|: t \in \boldsymbol{R}^{+}\right\} \leqq 1$, the desired conclusion follows from the first case.

$(\mathrm{VI})_{1} \Rightarrow(\mathrm{IV})_{1}$. This implication is obtained from the following:

$$
\begin{aligned}
& \sup _{r \geq 0}|B \cap(r, r+t]|^{-1}\left\|\int_{B \cap\langle r, r+t]} T(s) x d s\right\| \\
& \leqq \sup _{\substack{\|x *\| 1 \\
r \geq 0}}|B \cap(r, r+t]|^{-1} \int_{r}^{r+t}\left|\left\langle T(s) x, x^{*}\right\rangle\right| d s \\
& \leqq\left(\inf _{r \geqq 0} t^{-1}|B \cap(r, r+t]|\right)^{-1} \sup _{\substack{\left\|r^{*}\right\| \leq 1 \\
r \geq 0}} t^{-1} \int_{r}^{r+t}\left|\left\langle T(s) x, x^{*}\right\rangle\right| d s .
\end{aligned}
$$

$(\mathrm{IV})_{1} \Rightarrow(\mathrm{V})_{1}$. Let $(\mathrm{V})_{1}$ be false. Then there exist $B \subset \boldsymbol{R}^{+}$with $\beta=L D(B)>0$, $\varepsilon>0$ and $t_{n} \uparrow \infty$ such that

$$
\left\|\int_{B \cap\left(0, t_{n}\right]} T(s) x d s\right\| \geqq \varepsilon\left|B \cap\left(0, t_{n}\right]\right|, \quad n \geqq 1 .
$$

Since $\liminf _{n \rightarrow \infty} t_{n}{ }^{-1}\left|B \cap\left(0, t_{n}\right]\right| \geqq \beta$, we may assume that $t_{n}^{-1}\left|B \cap\left(0, t_{n}\right]\right| \geqq \beta / 2$ for all $n$. Let $0<\delta<\varepsilon \beta /(2 M\|x\|)$ and $C=B \cup \bigcup_{k=1}^{\infty}(k, k+\delta]$. Then we have $L D_{*}(C)>0$ and

$$
\begin{aligned}
& \left\|\int_{C \cap\left(0, t_{n}\right]} T(s) x d s\right\| \\
& \quad \geqq\left\|\int_{B \cap\left(0, t_{n}\right]} T(s) x d s\right\|-\left\|\int_{(C \backslash B) \cap\left(0, t_{n}\right]} T(s) x d s\right\|
\end{aligned}
$$




$$
\begin{aligned}
& \geqq \varepsilon\left|B \cap\left(0, t_{n}\right]\right|-M\|x\| \delta t_{n} \\
& \geqq\left(\frac{\varepsilon \beta}{2}-M\|x\| \delta\right) t_{n} \\
& \geqq\left(\frac{\varepsilon \beta}{2}-M\|x\| \delta\right)\left|C \cap\left(0, t_{n}\right]\right|,
\end{aligned}
$$

which contradicts $(\mathrm{IV})_{1}$.

$(\mathrm{VII})_{1} \Rightarrow(\mathrm{VIII})_{1}$. Let $x_{1}^{*}, \cdots, x_{n}^{*} \in E^{*}$ and $\varepsilon>0$ be given. Then (VII $)_{1}$ implies that

$$
\lim _{t \rightarrow \infty} t^{-1} \int_{0}^{t} \sum_{\imath=1}^{n}\left|\left\langle T(s) x, x_{\imath}^{*}\right\rangle\right| d s=0
$$

Thus there exists an $s \in \boldsymbol{R}^{+}$such that $\sum_{i=1}^{n}\left|\left\langle T(s) x, x_{i}^{*}\right\rangle\right|<\varepsilon$. This shows that (VIII $)_{1}$ holds. Consequently the statement $\left(1^{\circ}\right)$ is proved.

To prove the statement $\left(2^{\circ}\right)$, let $X$ be the weak closure of $\{T(t) x: t \geqq r\}$ where $r>0$, and assume $X$ to be metrizable in the weak topology. We need only show that (VIII) $)_{1} \Rightarrow(\mathrm{I})_{1}$. Let (VIII) $)_{1}$ hold. Then there exists a sequence $\left\{t_{n}\right\} \subset \boldsymbol{R}^{+}$such that $T\left(t_{n}\right) x \rightarrow 0$ (weakly). Assuming that sup $\left\{\|T(t)\|: t \in \boldsymbol{R}^{+}\right\} \leqq 1$, we define a one parameter semigroup $\left\{\hat{T}(t): t \in \boldsymbol{R}^{+}\right\}$on $C(K)$ as in the proof of $(\mathrm{VII})_{1} \Rightarrow(\mathrm{VI})_{1}$. Let $f\left(x^{*}\right)=\left|\left\langle x, x^{*}\right\rangle\right|$. Since $\hat{T}\left(t_{n}\right) f \rightarrow 0$ (weakly) in $C(K)$, it follows that $\langle f, \mu\rangle=0$ for every $\mu \in C(K)^{*}$ satisfying $\hat{T}(t)^{*} \mu=\mu, t \in \boldsymbol{R}^{+}$. Hence we see as before that $(\mathrm{VI})_{1}$ hold, so that (VII) $)_{1}$ holds. By the assumption, there exists a sequence $\left\{x_{k}^{*}\right\} \subset E^{*}$ such that $x_{\jmath} \rightarrow 0$ (weakly) in $X$ if and only if $\left\langle x_{\jmath}, x_{k}^{*}\right\rangle \rightarrow 0$ for all $k$. Since $t^{-1} \int_{0}^{t}\left|\left\langle T(s) x, x_{k}^{*}\right\rangle\right| d s \rightarrow 0$ for each $k$, using Lemma 1.2, we conclude that $(\mathrm{I})_{1}$ holds. Finally, if $\{T(t) x: t \geqq r\}$ is weakly sequentially compact, i. e., $X$ is weakly compact, then it follows from Lemma 2.3 that $X$ is metrizable in the weak topology. Thus $\left(2^{\circ}\right)$ is proved.

Q. E. D.

\section{§ 3. Semigroups on Banach spaces (2).}

As before, let $E$ be a Banach space. For $T \in \mathcal{L}(E)$, we consider the following conditions :

(i) $)_{2}$ there exists $a Q \in \mathcal{L}(E)$ such that for each $x \in E, T^{n} x$ converges weakly to $Q x$ as $n \in J, n \rightarrow \infty$ for some $J \subset Z^{+}$(dependent on $x$ ) with $d(J)=1$;

(ii) $)_{2}$ there exists a $Q \in \mathcal{L}(E)$ such that for each matrix $\left(\alpha_{n_{k}}\right)$ with (1.1), (1.2) and (1.4), $\sum_{k=1}^{\infty} \alpha_{n k} T^{k}$ converges in the strong operator topology to $Q$ as $n \rightarrow \infty$;

(iii) $)_{2}$ there exists a $Q \in \mathcal{L}(E)$ such that for each matrix $\left(\alpha_{n k}\right)$ with (1.1), (1.2) and (1.3), $\sum_{k=1}^{\infty} \alpha_{n k} T^{k}$ converges in the strong operator topology to $Q$ as $n \rightarrow \infty$; 
(iv) $)_{2}$ there exists a $Q \in \mathcal{L}(E)$ such that for each $K \subset Z^{+}$with $l d_{*}(K)>0$, the averages

$$
|K \cap\{\jmath+1, \cdots, \jmath+n\}|^{-1} \sum_{k \in K \cap(\jmath+1, \cdots, j+n\}} T^{k}
$$

converge in the strong operator topology to $Q$ as $n \rightarrow \infty$ uniformly with respect to $j \geqq 0$;

$(\mathrm{v})_{2}$ there exists $a Q \in \mathcal{L}(E)$ such that for each $K \subset Z^{+}$with $l d(K)>0$, the averages

$$
|K \cap\{1, \cdots, n\}|^{-1} \sum_{k \in K \cap\{1, \cdots, n\}} T^{k}
$$

converge in the strong operator topology to $Q$ as $n \rightarrow \infty$;

(vi) ${ }_{2}$ there exists a $Q \in \mathcal{L}(E)$ such that

$$
\lim _{n \rightarrow \infty} \sup _{\substack{\|x *\| \leqslant 1 \\ j \geq 0}} n^{-1} \sum_{k=j+1}^{j+n}\left|\left\langle\left(T^{k}-Q\right) x, x^{*}\right\rangle\right|=0
$$

for all $x \in E$;

(vii) $)_{2}$ there exists $a Q \in \mathcal{L}(E)$ such that

$$
\lim _{n \rightarrow \infty} n^{-1} \sum_{k=1}^{n}\left|\left\langle\left(T^{k}-Q\right) x, x^{*}\right\rangle\right|=0
$$

for all $x \in E$ and $x^{*} \in E^{*}$;

(viii) ${ }_{2}$ the closure of $\left\{T^{n}: n \in Z^{+}\right\}$in the weak operator topology contains a $Q \in \mathcal{L}(E)$ such that $T Q=Q$.

Let $\left\{T(t): t \in \boldsymbol{R}^{+}\right\}$be a strongly measurable and uniformly bounded semigroup in $\mathcal{L}(E)$. For a Lebesgue integrable real function $\phi$ on $\boldsymbol{R}^{+}$, the integral $\int_{0}^{\infty} \phi(t) T(t) d t \in \mathcal{L}(E)$ is defined by $\left(\int_{0}^{\infty} \phi(t) T(t) d t\right) x=\int_{0}^{\infty} \phi(t) T(t) x d t$ for $x \in E$. In particular, for a bounded set $A \subset \boldsymbol{R}^{+}$, the integral $\int_{A} T(t) d t \in \mathcal{L}(E)$ is defined. We then consider the following conditions:

$(\mathrm{I})_{2}$ there exists a $Q \in \mathcal{L}(E)$ such that for each $x \in E, T(t) x$ converges weakly to $Q x$ as $t \in A, t \rightarrow \infty$ for some $A \subset \boldsymbol{R}^{+}$(dependent on $x$ ) with $D(A)=1$;

$(\mathrm{II})_{2}$ there exists $a \in \in \mathcal{L}(E)$ such that for each sequence $\left\{\phi_{n}\right\}$ with (1.5), (1.6) and (1.8), $\int_{0}^{\infty} \phi_{n}(s) T(s) d s$ converges in the strong operator topology to $Q$ as $n \rightarrow \infty$;

$(\mathrm{III})_{2}$ there exists a $Q \in \mathcal{L}(E)$ such that for each sequence $\left\{\phi_{n}\right\}$ with (1.5), (1.6) and (1.7), $\int_{0}^{\infty} \phi_{n}(s) T(s) d s$ converges in the strong operator topology to $Q$ as $n \rightarrow \infty$;

$(\mathrm{IV})_{2}$ there exists a $Q \in \mathcal{L}(E)$ such that for each $B \subset \boldsymbol{R}^{+}$with $L D_{*}(B)>0$, the averages

$$
|B \cap(r, r+t]|^{-1} \int_{B \cap(r, r+t)} T(s) d s
$$


converge in the strong operator topology to $Q$ as $t \rightarrow \infty$ uniformly with respect to $r \geqq 0$;

$(\mathrm{V})_{2}$ there exists a $Q \in \mathcal{L}(E)$ such that for each $B \subset \boldsymbol{R}^{+}$with $L D(B)>0$, the averages

$$
|B \cap(0, t]|^{-1} \int_{B \cap(0, t]} T(s) d s
$$

converge in the strong operator topology to $Q$ as $t \rightarrow \infty$;

$(\mathrm{VI})_{2}$ there exists a $Q \in \mathcal{L}(E)$ such that

$$
\lim _{t \rightarrow \infty} \sup _{\substack{\|x *\| \leq 1 \\ r \geq 0}} t^{-1} \int_{r}^{r+t}\left|\left\langle(T(s)-Q) x, x^{*}\right\rangle\right| d s=0
$$

for all $x \in E$;

$(\mathrm{VII})_{2}$ there exists a $Q \in \mathcal{L}(E)$ such that

$$
\lim _{t \rightarrow \infty} t^{-1} \int_{0}^{t}\left|\left\langle(T(s)-Q) x, x^{*}\right\rangle\right| d s=0
$$

for all $x \in E$ and $x^{*} \in E^{*}$;

(VIII) $)_{2}$ the closure of $\left\{T(t): t \in \boldsymbol{R}^{+}\right\}$in the weak operator topology contains a $Q \in \mathcal{L}(E)$ such that $T(t) Q=Q$ for all $t \in \boldsymbol{R}^{+}$.

We now obtain the following:

Theorem 3.1. Let $T \in \mathcal{L}(E)$ and assume that $\sup \left\{\left\|T^{n}\right\|: n \in Z^{+}\right\}<\infty$. Then the following statements $\left(1^{\circ}\right)$ and $\left(2^{\circ}\right)$ hold:

$\left(1^{\circ}\right) \quad(\mathrm{i})_{2} \Rightarrow(\mathrm{ii})_{2} \Rightarrow(\mathrm{iii})_{2} \Rightarrow(\mathrm{iv})_{2} \Leftrightarrow(\mathrm{v})_{2} \Leftrightarrow(\mathrm{vi})_{2} \Leftrightarrow(\mathrm{vii})_{2} \Rightarrow(\mathrm{viii})_{2}$.

$\left(2^{\circ}\right)$ If for every $x \in E$, the weak closure of $\left\{T^{n} x: n \in \boldsymbol{Z}^{+}\right\}$is metrizable in the weak topology (in particular, if $\left\{T^{n}: n \in Z^{+}\right\}$is weakly almost periodic, i.e., relatively compact in the weak operator topology), then all the conditions $(\mathrm{i})_{2}-(\text { viii })_{2}$ are equivalent.

THEOREM 3.2. Let $\left\{T(t): t \in \boldsymbol{R}^{+}\right\}$be a strongly measurable and unformly bounded semigroup in $\mathcal{L}(E)$. Then the following statements $\left(1^{\circ}\right)$ and $\left(2^{\circ}\right)$ hold:

$\left(1^{\circ}\right) \quad(\mathrm{I})_{2} \Rightarrow(\mathrm{II})_{2} \Rightarrow(\mathrm{III})_{2} \Rightarrow(\mathrm{IV})_{2} \Leftrightarrow(\mathrm{V})_{2} \Leftrightarrow(\mathrm{VI})_{2} \Leftrightarrow(\mathrm{VII})_{2} \Rightarrow(\mathrm{VIII})_{2}$.

$\left(2^{\circ}\right)$ If for every $x \in E$, the weak closure of $\{T(t) x: t \geqq r\}$ is metrizable in the weak topology for some $r>0$ (in particular, if $\{T(t): t \geqq r\}$ is weakly almost periodic for some $r>0)$, then all the conditions $(\mathrm{I})_{2}-(\mathrm{VIII})_{2}$ are equivalent.

Theorems 3.1 and 3.2 are proved immediately from Theorems 2.1 and 2.2, respectively. Indeed, let $\left\{T(t): t \in \boldsymbol{R}^{+}\right\}$be as above. If one of the conditions $(\mathrm{I})_{2}-(\mathrm{VII})_{2}$ holds with $Q \in \mathcal{L}(E)$, then $Q$ is contained in the closure of $\left\{T(t): t \in \boldsymbol{R}^{+}\right\}$ in the weak operator topology and $T(t) Q=Q$ for all $t \in \boldsymbol{R}^{+}$. Thus, applying Theorem 2.2 with $x-Q x$ for each $x \in E$, we can prove Theorem 3.2 immediately.

REMARKS. (1) If $E$ is separable, then the conditions $(\mathrm{i})_{2}$ and $(\mathrm{I})_{2}$ may be replaced by the following $\left(\mathrm{i}^{\prime}\right)_{2}$ and $\left(\mathrm{I}^{\prime}\right)_{2}$, respectively: 


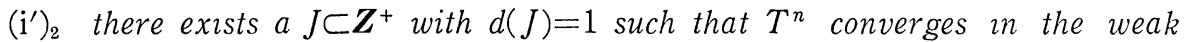
operator topology to a $Q \in \mathcal{L}(E)$ as $n \in J, n \rightarrow \infty$,

$\left(\mathrm{I}^{\prime}\right)_{2}$ there exists an $A \subset \boldsymbol{R}^{+}$with $D(A)=1$ such that $T(t)$ converges in the weak operator topology to a $Q \in \mathcal{L}(E)$ as $t \in A, t \rightarrow \infty$.

(2) Nagel $[20, \S 2]$ called $\left\{T^{n}: n \in Z^{+}\right\}$extremely ergodic if $T$ satisfies (viii) and showed that the conditions such as $\left(\mathrm{i}^{\prime}\right)_{2},(\mathrm{v})_{2},(\mathrm{vii})_{2}$ and (viii) $)_{2}$ are equivalent under the assumption of $\left\{T^{n}: n \in \boldsymbol{Z}^{+}\right\}$being metrizable and relatively compact in the weak operator topology. However the metrizability assumption is redundant for the equivalence of $(\mathrm{v})_{2},(\mathrm{vii})_{2}$, (viii) $)_{2}$ (and also $\left(\mathrm{i}^{\prime}\right)_{2}$ if $E$ is separable), as is stated in Theorem 3.1.

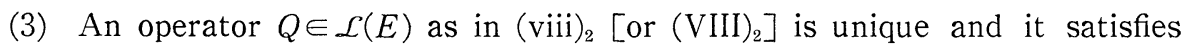
$Q^{2}=Q$ and $Q T=Q$ [or resp., $Q T(t)=Q$ for all $t \in \boldsymbol{R}^{+}$.

(4) If every separable subspace of $E$ has a separable dual, then the metrizability hypotheses in $\left(2^{\circ}\right)$ of Theorems 3.1 and 3.2 hold. According to Stegall [22], this is the case if and only if $E^{*}$ has the Radon-Nikodym property.

In the remainder of this section, we shall present some additional results concerning operator convergence of weak mixing type.

Theorem 3.3. Let $\left\{T(t): t \in \boldsymbol{R}^{+}\right\}$be as in Theorem 3.2, and let $r \in \boldsymbol{R}^{+}$. Then $\left\{T(t): t \in \boldsymbol{R}^{+}\right\}$satısfies the condition (VII) $)_{2}$ if and only if the following two conditions hold:

(1) if $T(r) x=x$, then $T(t) x=x$ for all $t \in \boldsymbol{R}^{+}$,

(2) $T(r)$ satısfies the condition (vii) $)_{2}$, i. e., there exists a $Q \in \mathcal{L}(E)$ such that

$$
\lim _{n \rightarrow \infty} n^{-1} \sum_{k=1}^{n}\left|\left\langle(T(k r)-Q) x, x^{*}\right\rangle\right|=0
$$

for all $x \in E$ and $x^{*} \in E^{*}$.

Proof. Assume that $\left\{T(t): t \in \boldsymbol{R}^{+}\right\}$satisfies (VII) $)_{2}$. To show (1), let $T(r) x=x$. Since

$$
\begin{aligned}
& (n r)^{-1} \int_{0}^{n r}\left|\left\langle(T(s)-Q) x, x^{*}\right\rangle\right| d s \\
& =(n r)^{-1} \sum_{k=1}^{n} \int_{0}^{r}\left|\left\langle(T(s) T((k-1) r)-Q) x, x^{*}\right\rangle\right| d s \\
& =(n r)^{-1} \sum_{k=1}^{n} \int_{0}^{r}\left|\left\langle(T(s)-Q) x, x^{*}\right\rangle\right| d s \\
& =r^{-1} \int_{0}^{r}\left|\left\langle(T(s)-Q) x, x^{*}\right\rangle\right| d s, \quad x^{*} \in E^{*}, n \geqq 1,
\end{aligned}
$$

we have $\left|\left\langle(T(s)-Q) x, x^{*}\right\rangle\right|=0$ on $(0, r]$ for all $x^{*} \in E^{*}$. Hence it follows that $T(s) x=Q x$ for $0<s \leqq r$, so that $T(t) x=x$ for all $t \in \boldsymbol{R}^{+}$. To show (2), let $\varepsilon>0$ be given. There exists a $\delta$ with $0<\delta<r$ such that $\|T(u) x-T(v) x\|<\varepsilon$ for $u, v \geqq r$, 
$|u-v|<\delta$. Then, for each $x^{*} \in E^{*}$ with $\left\|x^{*}\right\| \leqq 1$, since

$$
\begin{aligned}
& \left|\left\langle(T(k r)-Q) x, x^{*}\right\rangle\right| \\
& \quad \leqq\left|\left\langle(T(k r+s)-Q) x, x^{*}\right\rangle\right|+\varepsilon, \quad 0 \leqq s<\delta, k \geqq 1,
\end{aligned}
$$

we have

$$
\begin{aligned}
& n^{-1} \sum_{k=1}^{n}\left|\left\langle(T(k r)-Q) x, x^{*}\right\rangle\right| \\
& \quad \leqq(n \delta)^{-1} \int_{0}^{(n+1) r}\left|\left\langle(T(s)-Q) x, x^{*}\right\rangle\right| d s+\varepsilon, \quad n \geqq 1 .
\end{aligned}
$$

Thus we have

$$
\limsup _{n \rightarrow \infty} n^{-1} \sum_{k=1}^{n}\left|\left\langle(T(k r)-Q) x, x^{*}\right\rangle\right| \leqq \varepsilon
$$

which shows (2).

Conversely, assume that (1) and (2) hold. Let $Q \in \mathcal{L}(E)$ be taken in (2). Then it follows from (1) that $T(t) Q=Q$ for all $t \in \boldsymbol{R}^{+}$. For each $x^{*} \in E^{*}$ and $n r<t \leqq(n+1) r$, we have

$$
\begin{aligned}
t^{-1} \int_{r}^{t} \mid & \left\langle(T(s)-Q) x, x^{*}\right\rangle \mid d s \\
& \leqq(n r)^{-1} \sum_{k=1}^{n} \int_{k r}^{(k+1) r}\left|\left\langle(T(s)-Q) x, x^{*}\right\rangle\right| d s \\
& =(n r)^{-1} \sum_{k=1}^{n} \int_{0}^{r}\left|\left\langle(T(k r)-Q) x, T(s)^{*} x^{*}\right\rangle\right| d s \\
& =r^{-1} \int_{0}^{r} n^{-1} \sum_{k=1}^{n}\left|\left\langle(T(k r)-Q) x, T(s)^{*} x^{*}\right\rangle\right| d s .
\end{aligned}
$$

Putting

$$
\phi_{n}(s)=n^{-1} \sum_{k=1}^{n}\left|\left\langle(T(k r)-Q) x, T(s)^{*} x^{*}\right\rangle\right|, \quad n \geqq 1,
$$

since $\left\{\phi_{n}\right\}$ is uniformly bounded and $\phi_{n}(s) \rightarrow 0$ on $(0, r]$, we obtain

$$
\lim _{t \rightarrow \infty} t^{-1} \int_{0}^{t}\left|\left\langle(T(s)-Q) x, x^{*}\right\rangle\right| d s=\lim _{n \rightarrow \infty} r^{-1} \int_{0}^{r} \phi_{n}(s) d s=0
$$

by Lebesgue's convergence theorem.

Q.E. D.

THEOREM 3.4. Let $\{T(t): t \in \boldsymbol{R}\}$ be a strongly measurable and unformly bounded group in $\mathcal{L}(E)$. Then $\left\{T(t): t \in \boldsymbol{R}^{+}\right\}$satisfies the condition (VII) $)_{2}$ if and only if $\left\{T(-t): t \in \boldsymbol{R}^{+}\right\}$does.

Proof. Assume that $\left\{T(t): t \in \boldsymbol{R}^{+}\right\}$satisfies (VII) $)_{2}$. Then it satisfies also $(\mathrm{VI})_{2}$ by Theorem 3.2. Thus, for each $x_{0}^{*} \in E^{*}$ with $\left\|x_{0}^{*}\right\| \leqq 1$, we have 


$$
\begin{aligned}
t^{-1} \int_{0}^{t} & \left\langle(T(-s)-Q) x, x_{0}^{*}\right\rangle \mid d s \\
& =t^{-1} \int_{0}^{t}\left|\left\langle T(-t)(T(t-s)-Q) x, x_{0}^{*}\right\rangle\right| d s \\
& =t^{-1} \int_{0}^{t}\left|\left\langle(T(s)-Q) x, T(-t)^{*} x_{0}^{*}\right\rangle\right| d s \\
& \leqq \sup _{\left\|x^{*}\right\| M} t^{-1} \int_{0}^{t}\left|\left\langle(T(s)-Q) x, x^{*}\right\rangle\right| d s \longrightarrow 0
\end{aligned}
$$

as $t \rightarrow \infty$, where $M=\sup \{\|T(t)\|: t \in \boldsymbol{R}\}$. Therefore $\left\{T(-t): t \in \boldsymbol{R}^{+}\right\}$satisfies $(\mathrm{VII})_{2}$. The converse is analogous. $\quad$ Q.E.D.

Also for discrete semigroups or groups in $\mathcal{L}(E)$, we can obtain the results analogous to Theorems 3.3 and 3.4 in the same way.

\section{§4. Semigroups on AL-spaces.}

In this section, let $E$ be an $A L$-space, i. e., $E=L_{1}(\Omega, \mu)$, the Banach space of (equivalence classes of) integrable functions on a measure space $(\Omega, \mathscr{F}, \mu)$. Let $\mathcal{L}_{+}(E)$ be the set of all positive operators in $\mathcal{L}(E)$. The following Theorems 4.1 and 4.2 have been shown in $[8, \S 3]$ for $E=L_{1}(\Omega, \mu)$ over a $\sigma$-finite measure space $(\Omega, \mathscr{F}, \mu)$. However these theorems are valid over a general measure space $(\Omega, \mathscr{F}, \mu)$. Indeed, for each $x \in E$, there exists a set $\Omega_{0} \in \mathscr{F}$ on which $\mu$ is $\sigma$-finite and such that $x \in L_{1}\left(\Omega_{0}, \mu\right)$ and $T L_{1}\left(\Omega_{0}, \mu\right) \subset L_{1}\left(\Omega_{0}, \mu\right)$ [or $T(t) L_{1}\left(\Omega_{0}, \mu\right)$ $\subset L_{1}\left(\Omega_{0}, \mu\right)$ for all $\left.t \in \boldsymbol{R}^{+}\right]$.

Theorem 4.1. Let $T \in \mathcal{L}_{+}(E)$ and assume that $\sup \left\{\left\|T^{n}\right\|: n \in Z^{+}\right\}<\infty$. Then the following conditions are equivalent:

(a) for each $x \in E$, the averages $n^{-1} \sum_{k=1}^{n} T^{k} x$ converge strongly as $n \rightarrow \infty$;

(b) for each $x \in E$, the closed convex hull of $\left\{T^{n} x: n \in \boldsymbol{Z}^{+}\right\}$contains an $x_{0} \in E$ such that $T x_{0}=x_{0}$;

(c) $\left\{T^{n}: n \in Z^{+}\right\}$is weakly almost periodic.

THEOREM 4.2. Let $\left\{T(t): t \in \boldsymbol{R}^{+}\right\}$be a strongly measurable and unformly bounded semigroup in $\mathcal{L}_{+}(E)$. Then the following conditions are equivalent:

(a) for each $x \in E$, the averages $t^{-1} \int_{0}^{t} T(s) x d s$ converge strongly as $t \rightarrow \infty$;

(b) for each $x \in E$, the closed convex hull of $\left\{T(t) x: t \in \boldsymbol{R}^{+}\right\}$contams an $x_{0} \in E$ such that $T(t) x_{0}=x_{0}$ for all $t \in \boldsymbol{R}^{+}$;

(c) $\{T(t): t \geqq r\}$ is weakly almost periodic for each $r>0$.

Combining Theorems 3.1 and 3.2 with Theorems 4.1 and 4.2 respectively, we have the following: 
THEOREM 4.3. Let $T \in \mathcal{L}_{+}(E)$ be as in Theorem 4.1. Then all the conditions $(\mathrm{i})_{2}-(\text { viii })_{2}$ are equivalent.

THEOREM 4.4. Let $\left\{T(t): t \in \boldsymbol{R}^{+}\right\} \subset \mathcal{L}_{+}(E)$ be as in Theorem 4.2. Then all the conditions $(\mathrm{I})_{2}-(\mathrm{VIII})_{2}$ are equivalent.

\section{REFERENCES}

[1] M. Akcoglu And L. Sucheston, On operator convergence in Hilbert space and in Lebesgue space, Periodica Math. Hungarica 2 (1972), 235-244.

[2] M. Akcoglu and L. Sucheston, On convergence of iterates of positive contractions in $L_{p}$ spaces, J. Approximation Theory 13 (1975), 348-362

[3] J.R. Blum and D.L. Hanson, On the mean ergodic theorem for subsequences, Bull. Amer. Math. Soc. 66 (1960), 308-311.

[4] A. Brunel And M. Keane, Ergodic theorems for operator sequences, $Z$. Wahrscheinlichkeitstheorie und Verw. Gebiete 12 (1969), 231-240.

[5] K. Deleeuw and I. Glicksberg, Applications of almost periodic compactifications, Acta Math. 105 (1961), 63-97.

[6] H. Fong And L. Sucheston, On a mixing property of operators in $L_{p}$ spaces, Z. Wahrscheinlichkeitstheorie und Verw. Gebiete 28 (1974), 165-171.

[7] D. L. HANSON ANd G. Pledger, On the mean ergodic theorem for weighted averages, $Z$. Wahrscheinlichkeitstheorie und Verw. Gebiete 13 (1969), 141-149.

[8] F. Hiai AND R. SAto, Mean ergodic theorems for semigroups of positive linear operators, J. Math. Soc. Japan 29 (1977), 123-134.

[9] E. Hille and R.S. Phillps, Functional Analysis and Semi-groups, Amer. Math. Soc. Colloq. Pub. Vol. 31, Providence, R. I., 1957.

[10] K. JACOBS, Neuere Methoden und Ergebnisse der Ergodentheorie, SpringerVerlag, Berlin-Göttingen-Heidelberg, 1960.

[11] L.K. JonEs, A mean ergodic theorem for weakly mixing operators, Advances in Math. 7 (1971), 211-216.

[12] L.K. Jones, An elementary lemma on sequences of integers and its applications to functional analysis, Math. Z. 126 (1972), 299-307.

[13] L.K. Jones, A generalization of the mean ergodic theorem in Banach spaces, Z. Wahrscheinlichke1tstheorie und Verw. Gebiete 27 (1973), 105-107.

[14] L.K. Jones And M. Lin, Ergodic theorems of weak mixing type, Proc. Amer. Math. Soc. 57 (1976), 50-52.

[15] H.D. JunghenN, Almost periodic compactifications and applications to oneparameter semigroups, Doctoral dissertation, The George Washington Univ., 1971.

[16] U. KRENGEL, Weakly wandering vectors and weakly independent partitions, Trans. Amer. Math. Soc. 164 (1972), 199-226.

[17] U. Krengel and L. Sucheston, On mixing in infinite measure spaces, $Z$. Wahrscheinlichkeitstheor1e und Verw. Gebiete 13 (1969), 150-164.

[18] M. Lin, Mixing for Markov operators, Z. Wahrscheınlichkeitstheorie und Verw. Gebiete 19 (1971), 231-242.

[19] R. J. NAGEL, Mittelergodische halbgruppen linearer operatoren, Ann. Inst. Fourier (Grenoble) 23-4 (1973), 75-87. 
[20] R. J. NAgel, Ergodic and mixing properties of linear operators, Proc. Roy. Irish Acad. Sect. A. 74 (1974), 245-261.

[21] R. S^ то, On Akcoglu and Sucheston's operator convergence theorem in Lebesgue space, Proc. Amer. Math. Soc. 40 (1973), 513-516.

[22] C. Stegall, The Radon-Nikodym property in conjugate Banach spaces, Trans. Amer. Math. Soc. 206 (1975), 213-223.

[23] W. TAKAHASHI, Invariant ideals for amenable semigroups of Markov operators, Kōdai Math. Sem. Rep. 23 (1971), 121-126.

[24] P. Walters, Ergodic Theory-Introductory Lectures, Springer-Verlag, Lecture Notes in Math., Vol. 458, 1975.

Department of Information Sciences

Faculty of Science and Technology

SCIENCE University of TOKYo

Noda, Chiba 278

JAPAN 